PROCEEDINGS OF THE

AMERICAN MATHEMATICAL SOCIETY

Volume 137, Number 5, May 2009, Pages 1723-1728

S 0002-9939(08)09816-X

Article electronically published on December 11, 2008

\title{
ON A CONJECTURE OF TOMAS SAUER REGARDING NESTED IDEAL INTERPOLATION
}

\author{
BORIS SHEKHTMAN
}

(Communicated by Nigel J. Kalton)

\begin{abstract}
Tomas Sauer conjectured that if an ideal complements polynomials of degree less than $n$, then it is contained in a larger ideal that complements polynomials of degree less than $n-1$. We construct a counterexample to this conjecture for polynomials in three variables and with $n=3$.
\end{abstract}

\section{INTRODUCTION AND PRELIMINARIES}

Let $\mathbb{C}\left[x_{1}, \ldots, x_{d}\right]$ denote the space of polynomials in $d$ variables with complex coefficients, let $\mathbb{C}_{<n}\left[x_{1}, \ldots, x_{d}\right]$ denote its subspace of polynomials of degree less than $n$ and let $\left(\mathbb{C}\left[x_{1}, \ldots, x_{d}\right]\right)^{\prime}:=\operatorname{Hom}_{\mathbb{C}}\left(\mathbb{C}\left[x_{1}, \ldots, x_{d}\right], \mathbb{C}\right)$ denote the algebraic dual of $\mathbb{C}\left[x_{1}, \ldots, x_{d}\right]$, i.e., the space of all linear functionals on $\mathbb{C}\left[x_{1}, \ldots, x_{d}\right]$.

Definition 1.1. Let $\Lambda$ be a subset of $\left(\mathbb{C}\left[x_{1}, \ldots, x_{d}\right]\right)^{\prime}$ and $E$ be a linear subspace of $\mathbb{C}\left[x_{1}, \ldots, x_{d}\right]$. We say that $\Lambda$ is correct for $E$ if for every $f \in \mathbb{C}\left[x_{1}, \ldots, x_{d}\right]$ there exists a unique $g \in E$ such that $\lambda(g)=\lambda(f)$ for every $\lambda \in \Lambda$.

With every subspace $\Lambda \subset\left(\mathbb{C}\left[x_{1}, \ldots, x_{d}\right]\right)^{\prime}$ we associate the subspace $\operatorname{ker} \Lambda \subset$ $\mathbb{C}\left[x_{1}, \ldots, x_{d}\right]$ defined by

$$
\operatorname{ker} \Lambda:=\left\{f \in \mathbb{C}\left[x_{1}, \ldots, x_{d}\right]: \lambda(f)=0 \text { for all } \lambda \in \Lambda\right\} .
$$

The purpose of this paper is to construct a counterexample to the following conjecture of Tomas Sauer:

Conjecture 1.2 ([8, Conj. 4.1]). Let $\Lambda$ be a subspace of $\left(\mathbb{C}\left[x_{1}, \ldots, x_{d}\right]\right)^{\prime}$ such that $\Lambda$ is correct for $\mathbb{C}_{<n}\left[x_{1}, \ldots, x_{d}\right]$ and $\operatorname{ker} \Lambda$ is an ideal in $\mathbb{C}\left[x_{1}, \ldots, x_{d}\right]$. Then there exists a subspace $\Lambda_{0} \subset \Lambda$ such that $\Lambda_{0}$ is correct for $\mathbb{C}_{<n-1}\left[x_{1}, \ldots, x_{d}\right]$ and ker $\Lambda_{0}$ is an ideal in $\mathbb{C}\left[x_{1}, \ldots, x_{d}\right]$.

Some specific spaces $\Lambda$ for which the conjecture is valid can also be found in 9 and 10. In particular, if $\operatorname{ker} \Lambda$ is a radical ideal, then the conjecture is verified. If $\operatorname{ker} \Lambda$ is radical, then the associated variety consists of the maximal possible number of points. The counterexample, presented in the next section, is constructed in the space of polynomials of three variables and with $n=3$. The corresponding ideal $\operatorname{ker} \Lambda$ is primary, hence is at the other extreme; i.e., the variety of ker $\Lambda$ consists of just one point.

Received by the editors May 30, 2008.

2000 Mathematics Subject Classification. Primary 41A63; Secondary 41A10, 41A80, 13P10.

Key words and phrases. Ideal interpolation, nested ideals, multivariate divided differences.

(C)2008 American Mathematical Society 
We will use the rest of this section to recall some well-known facts regarding duality (inverse systems) for $\mathbb{C}\left[x_{1}, \ldots, x_{d}\right]$.

We will identify the space $\left(\mathbb{C}\left[x_{1}, \ldots, x_{d}\right]\right)^{\prime}$ with the space $\mathbb{C}\left[\left[x_{1}, \ldots, x_{d}\right]\right]$ of formal power series as follows:

With every element $F \in \mathbb{C}\left[\left[x_{1}, \ldots, x_{d}\right]\right]$ we associate a differential operator $F(D) \in \mathbb{C}\left[D_{1}, \ldots, D_{d}\right]$ by formally replacing variables in $F$ with the appropriate powers of operators $D_{j}$ which are partial derivatives with respect to $x_{j}$. Now, for every $F \in \mathbb{C}\left[\left[x_{1}, \ldots, x_{d}\right]\right]$ we define the functional $\hat{F} \in\left(\mathbb{C}\left[x_{1}, \ldots, x_{d}\right]\right)^{\prime}$ by

$$
\hat{F}(f):=(F(D) f)(0) \text { for every } f \in \mathbb{C}\left[x_{1}, \ldots, x_{d}\right] .
$$

It is well-known (cf. [3, [5] and [6]) that the map $F \longmapsto \hat{F}$ defined by (1.1) is a skew-linear isomorphism between $\mathbb{C}\left[\left[x_{1}, \ldots, x_{d}\right]\right]$ and $\left(\mathbb{C}\left[x_{1}, \ldots, x_{d}\right]\right)^{\prime}$. If $M \subset$ $\mathbb{C}\left[\left[x_{1}, \ldots, x_{d}\right]\right]$ we use $\operatorname{ker} M$ to denote the space $\operatorname{ker} \hat{M}$, i.e.,

$$
\operatorname{ker} M:=\left\{f \in \mathbb{C}\left[x_{1}, \ldots, x_{d}\right]: \hat{F}(f)=0 \text { for all } F \in M\right\} \text {. }
$$

Definition 1.3. A subspace $M \subset \mathbb{C}\left[\left[x_{1}, \ldots, x_{d}\right]\right]$ is called $D$-invariant if for every $F \in M$,

$$
D_{j} F \in M \text { for all } j=1, \ldots, d \text {. }
$$

Theorem 1.4 (cf. [3], $[6$ and $[5$ in its original form). Let $M$ be a finite-dimensional subspace of $\mathbb{C}\left[\left[x_{1}, \ldots, x_{d}\right]\right]$. Then $\operatorname{ker} M$ is an ideal in $\mathbb{C}\left[x_{1}, \ldots, x_{d}\right]$ if and only if $M$ is D-invariant.

In this terminology, to construct a counterexample to Conjecture 1.2, we need to construct a $D$-invariant subspace $M \subset \mathbb{C}\left[\left[x_{1}, \ldots, x_{d}\right]\right]$ such that $M$ is correct for $\mathbb{C}_{<n}\left[x_{1}, \ldots, x_{d}\right]$ while no $D$-invariant subspace $N \subset M$ is correct for $\mathbb{C}_{<n-1}\left[x_{1}, \ldots, x_{d}\right]$.

As a warm-up, consider the following simple example, which already gives a negative answer to Conjecture 5.14 of $[8]$ in two variables.

Example 1. Let $E$ be the subspace of $\mathbb{C}[x, y]$ spanned by the four polynomials

$$
f_{1}=1, f_{2}=x, f_{3}=y, f_{4}=x^{2} .
$$

Since $\hat{f}_{j}\left(f_{k}\right) \neq 0$ if and only if $j \neq k$ it follows that $\hat{E}$ is correct for $E$. Modifying $E$ by replacing $f_{3}$ with $F_{3}:=f_{3}+x^{3}$ leaves the action $\hat{F}_{3}$ on $E$ unchanged and also preserves $D$-invariance. Hence the subspace $M \subset \mathbb{C}[[x, y]]$ spanned by

$$
F_{1}=1, F_{2}=x, F_{3}=y+x^{3}, F_{4}=x^{2}
$$

is also $D$-invariant and correct for $E$. On the other hand, no three-dimensional $D$-invariant subspace $N \subset M$ is correct for the space $E_{1}:=\operatorname{span}\{1, x, y\}$. Indeed, by virtue of being $D$-invariant and three-dimensional, $N$ could not contain a polynomial of degree 3 for, if it did, the consecutive partial derivatives of such a polynomial would span a four-dimensional subspace. Hence $N=\operatorname{span}\left\{1, x, x^{2}\right\}$ is the only three-dimensional $D$-invariant subspace of $M$. This space is not correct for $E_{1}$ since every functional associated with a polynomial in $N$ vanishes on $y \in E$. 


\section{Counterexample to Conjecture 1.2}

Conjecture 1.2 is slightly more restrictive in that it only deals with ideal projectors whose range is $\mathbb{C}_{<n}\left[x_{1}, \ldots, x_{d}\right]$ for some $n$; hence Example 1 does not contradict it. But expanding on the last example, we will now describe a $D$-invariant 10-dimensional subspace $M \subset \mathbb{C}[[x, y, z]]$ that is correct for $\mathbb{C}_{<3}[x, y, z]$, such that no 4-dimensional $D$-invariant subspace $N \subset M$ is correct for $\mathbb{C}_{<2}[x, y, z]$.

We again start with the basis

$$
\begin{gathered}
f_{1}=1, f_{2}=x, f_{3}=x^{2}, f_{4}=y, f_{5}=x y \\
f_{6}=z, f_{7}=z^{2}, f_{8}=x z, f_{9}=y^{2}, f_{10}=y z
\end{gathered}
$$

for the space $E:=\mathbb{C}_{<3}[x, y, z]$ and introduce the polynomials $F_{j}(j=1, \ldots, 10)$ as higher-order perturbations of $f_{j}$ :

$$
\begin{aligned}
F_{1} & =1, F_{2}=x, F_{3}=x^{2}, F_{4}=y+x^{3}, \\
F_{5} & =x y+\frac{1}{4} x^{4}, F_{6}=z, F_{7}=z^{2}, \\
F_{8} & =x z+\frac{1}{2} x^{2} y+\frac{1}{20} x^{5}, \\
F_{9} & =y^{2}+2 x^{3} y+\frac{1}{10} x^{6}+6 x^{2} z, \\
F_{10} & =z y+\frac{1}{140} x^{7}+\frac{1}{4} x^{4} y+z x^{3}+\frac{1}{2} x y^{2} .
\end{aligned}
$$

Since $F_{j}$ has the same action on $E$ as $f_{j}$, the space $M \subset \mathbb{C}[[x, y, z]]$ spanned by the 10 polynomials $F_{1}, \ldots, F_{10}$ is correct for $E$.

The verification that $M$ is $D$-invariant is by direct computations whose results are presented in the table below:

$$
\left(D_{j}\left(F_{i}\right): i=1, \ldots, 10, j=1,2,3\right)=\left[\begin{array}{l|l|l}
0 & 0 & 0 \\
F_{1} & 0 & 0 \\
2 F_{2} & 0 & 0 \\
3 F_{3} & F_{1} & 0 \\
F_{4} & F_{2} & 0 \\
0 & 0 & F_{1} \\
0 & 0 & 2 F_{6} \\
F_{5}+F_{6} & \frac{1}{2} F_{3} & F_{2} \\
12 F_{8} & 2 F_{4} & 6 F_{3} \\
\frac{1}{2} F_{9} & F_{5}+F_{6} & F_{4}
\end{array}\right] .
$$

It remains to prove that no $D$-invariant subspace $N \subset M$ is correct for

$$
\mathbb{C}_{<2}[x, y, z]=\operatorname{span}\{1, x, y, z\} .
$$

Proof. Observe that if $N$ is correct for $\mathbb{C}_{<2}[x, y, z]$, then $N$ is 4-dimensional; and hence if it is also $D$-invariant, then it cannot contain polynomials of degree 4 or larger, since the partial derivatives of a polynomial of degree $k$ span a subspace of dimension $\geq k+1$. Since the polynomials $F_{5}, F_{8}, F_{9}, F_{10}$ are of different total degree all $>3$, any correct $D$-invariant $N$ must be in the linear span of the other $F_{i}$, i.e.,

$$
F_{1}=1, F_{2}=x, F_{3}=x^{2}, F_{4}=y+x^{3}, F_{6}=z, F_{7}=z^{2} .
$$


Now assume that $N$ is correct for $\mathbb{C}_{<2}[x, y, z]$. Then $N$ contains a polynomial $F$ for which

$$
\hat{F}(y)=1, \quad \hat{F}(1)=\hat{F}(x)=\hat{F}(z)=0 .
$$

Such a polynomial must be of the form

$$
F=F_{4}+a F_{3}+b F_{7}
$$

for some constants $a$ and $b$, for, if it were to contain a nonzero multiple of any other polynomial in (2.1), then at least one of the last three equalities in (2.2) would fail. By $D$-invariance,

$$
D_{1} F=3 x^{2}+2 a x \in N,
$$

and by repeated differentiation, 1 and $x$ belong to $N$. Since $N$ is four-dimensional, it follows that

$$
N=\operatorname{span}\left\{1, x, x^{2}, F=x^{3}+y+\frac{a}{2} x^{2}+\frac{b}{2} z^{2}\right\}
$$

hence ker $\hat{N}$ contains $z \in \mathbb{C}_{<2}[x, y, z]$. This contradicts the assumption that $N$ is correct for $\mathbb{C}_{<2}[x, y, z]$.

\section{Discussion}

In this section we wish to discuss some implications that the above example has in the theory of ideal interpolation and the Newton form for multivariate ideal projectors.

Definition 3.1 (G. Birkhoff, [1]). A linear idempotent operator $P$ on $\mathbb{C}\left[x_{1}, \ldots, x_{d}\right]$ is called an ideal projector if $\operatorname{ker} P$ is an ideal in $\mathbb{C}\left[x_{1}, \ldots, x_{d}\right]$.

Recall that an ideal projector is called a Lagrange projector if $P f$ is the unique element in its range that agrees with $f$ at a certain finite set $Z$ in $\mathbb{C}^{d}$. For its kernel consists of exactly those polynomials that vanish on $Z$; i.e., it is the zerodimensional radical ideal $J(Z)$.

An ideal projector $P$ is called a Hermite projector if it is the limit of Lagrange projectors, i.e., if there exists a sequence of Lagrange projectors $P_{m}$ onto the range of $P$ such that $P_{n} f \rightarrow P f$ for every $f \in \mathbb{C}\left[x_{1}, \ldots, x_{d}\right]$.

An ideal projector onto $\mathbb{C}_{<n}\left[x_{1}, \ldots, x_{d}\right]$ is completely determined by its values on monomials of degree $n$ (cf. [2]). That is, the set

$$
\{u-P u: u \text { is a monomial of degree } n\}
$$

is a basis for the ideal ker $P$.

Let $M$ be the space of polynomials constructed in the previous section. Since ker $\hat{M}$ complements $\mathbb{C}_{<3}[x, y, z]$, it gives rise to the linear projector $P$ with range $\mathbb{C}_{<3}[x, y, z]$ and kernel ker $\hat{M}$; hence $P$ is ideal. By direct computation,

$$
\begin{gathered}
P x^{3}=6 y, P x^{2} y=x z, P x y^{2}=z y, P x^{2} z=6 y^{2}, \\
P x z^{2}=P z^{3}=P z^{2} y=P z y^{2}=P y^{3}=0
\end{gathered}
$$

hence

(3.2) $\operatorname{ker} P=\operatorname{ideal}\left(x^{3}-6 y, x^{2} y-x z, x y^{2}-z y, x^{2} z-6 y^{2}, x z^{2}, z^{3}, z^{2} y, z y^{2}, y^{3}\right)$.

Since Lagrange projectors are defined by radical ideals and Hermite projectors are the limits of Lagrange ones, one might expect that Conjecture 1.2, being true 
for radical ideals, implies its validity for the limits of those ideals. Unfortunately this is not the case.

Proposition 3.2. The ideal projector $P$ onto $\mathbb{C}_{<3}[x, y, z]$ given by (3.1) is Hermite.

Proof. Using (3.1) we can compute explicitly the matrices of commuting multiplication operators $\mu_{x}, \mu_{y}, \mu_{z}$ defined on $\mathbb{C}_{<3}[x, y, z]$ as

$$
\mu_{v}(f):=P(v f), v=x, y, z .
$$

Direct computations show that the nilpotent operator $\mu_{x}+\mu_{z}$ is 2-regular and its eigenspace is two-dimensional, spanned by the polynomials $z^{2}$ and $y z$. As the three operators in (3.3) commute with the 2-regular operator $\mu_{x}+\mu_{z}$, they can be approximated by commuting simultaneously diagonalizable operators (cf. 7 , Theorem 6.1]). This, in turn, implies (cf. [4]) that $P$ is a Hermite projector.

It follows that there does not exist a "continuous" Newton form for multivariate interpolation, hence a "continuous" notion of multivariate divided differences. Indeed, suppose that $P_{m}^{(3)}$ are Lagrange projectors onto $\mathbb{C}_{<3}[x, y, z]$ written in Newton form:

$$
P_{m}^{(3)} f=P_{m}^{(2)} f+R_{m} f,
$$

where $P_{m}^{(2)}$ are Lagrange projectors onto $\mathbb{C}_{<2}[x, y, z]$ that interpolate at some appropriately chosen (such as in [10]) subset of interpolation sites of $P_{m}^{(3)}$ and the $R_{m}$ are linear operators constructed via an appropriately defined "divided differences" at those sites. Then, if $P_{m}^{(3)}$ converges to $P$ defined in (3.1), the left-hand side of (3.4) converges to a limit while neither $P_{m}^{(2)}$ nor $R_{m}$ converges.

\section{ACKNOWLEDGMENT}

I would like to express my gratitude to Carl de Boor for many useful suggestions.

\section{REFERENCES}

1. G. Birkhoff, The algebra of multivariate interpolation, Constructive approaches to mathematical models (Proc. Conf. in honor of R. J. Duffin, Pittsburgh, Pa., 1978) (C. V. Coffman and G. J. Fix, eds.), Academic Press, New York, NY, 1979, pp. 345-363. MR.559505 (83d:41001)

2. C. de Boor, Ideal interpolation, Approximation Theory XI: Gatlinburg, 2004 (C. K. Chui, M. Neamtu, and L. Schumaker, eds.), Mod. Methods Math., Nashboro Press, Brentwood, TN, 2005, pp. 59-91. MR2126674 (2005k:41004)

3. C. de Boor and A. Ron, On polynomial ideals of finite codimension with applications to box spline theory, J. Math. Anal. Appl. 158 (1991), no. 1, 168-193. MR.1113408 (93a:41014)

4. C. de Boor and B. Shekhtman, On the pointwise limits of bivariate Lagrange projectors, Linear Algebra Appl. 429 (2008), no. 1, 311-325. MR2419159

5. F. S. Macaulay, The algebraic theory of modular systems, Cambridge Mathematical Library, Cambridge University Press, Cambridge, 1994, revised reprint of the 1916 original, with an introduction by Paul Roberts. MR.1281612 (95i:13001)

6. Hans Michael Möller, Hermite interpolation in several variables using ideal-theoretic methods, Constructive theory of functions of several variables (Proc. Conf., Math. Res. Inst., Oberwolfach, 1976), Lecture Notes in Math., vol. 571, Springer, Berlin, 1977, pp. 155-163. MR0493046 $(58: 12087)$

7. K. C. O'Meara and C. Vinsonhaler, On approximately simultaneously diagonalizable matrices, Linear Algebra Appl. 412 (2006), no. 1, 39-74. MR2180858 (2006g:15022) 
8. T. Sauer, Polynomial interpolation in several variables: Lattices, differences, and ideals, Topics in Multivariate Approximation and Interpolation (M. Buhmann, W. Hausmann, K. Jetter, R. Schaback, and J. Stöckler, eds.), Studies in Computational Mathematics, vol. 12, Elsevier, Amsterdam, The Netherlands, 2005, pp. 189-228.

9. T. Sauer and Yuan Xu, On multivariate Hermite interpolation, Advances Comput. Math. 4 (1995), no. 4, 207-259. MR1357718 (96j:41031)

10. - On multivariate Lagrange interpolation, Math. Comp. 64 (1995), 1147-1170. MR.1297477 (95j:41051)

Department of Mathematics and Statistics, University of South Florida, Tampa, FLORIDA 33620

E-mail address: boris@math.usf.edu

URL: http://www.math.usf.edu/ boris/ 